92 Thode: 0-Amidobenzhydrazid u. Abkőmml. desselben.

\title{
Untersuchungen aus dem organischen Laboratorium der Technischen Hochschule zu Dresden.
}

\section{XVIII. 0-Amidobenzhydrazid und Abkömmlinge desselben; \\ ron \\ C. Thode. ${ }^{1}$ )}

Bei der Erforschung des 0-A midobenzhydrazids kommt auBer seiner Hydrazidnatur der Einfluß der Orthostellung der Amidogruppe auf sein chemisches Verhalten stark in Frage.

Zur Gewinnung des o-Amidobenzhydrazids diente Isatosäure in der gleichen Weise, wie früher von E. v. Meyer und Th. Bellmann') aus derselben mittels Phenylhydrazin das 0-A midobenzphenylhydrazid dargestellt worden war.

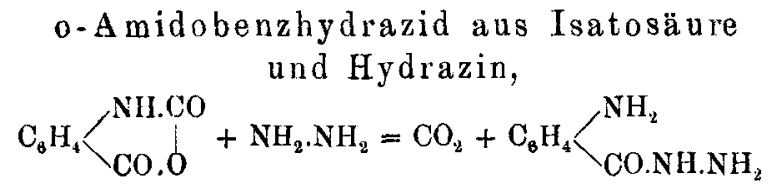

Die zur Gewinnung des Bydrazids erforderliche Isatosäure wurde nach dem trefflichen Erdmannschen Verfahren ${ }^{3}$ ) gewonnen. In die Lösung von $50 \mathrm{~g}$ Anthranilsäure und $20 \mathrm{~g}$ kohlensaurem Natrium in 11 Wasser leitet man Phosgengas ein; sofort entsteht ein gelber Niederschlag, während die Reaktion der Lösung sauer wird. Allmählich gibt man noch $20 \mathrm{~g}$ Natriumkarbonat in 5 prozent. Lösung hinzu und achtet darauf, dak die Lösung immer sauer bleibt. Das Phosgengas wird anfangs vollständig absorbiert, zum SchluB weniger vollständig, so dab man gut tut, eine zweite Flasche mit aufgelöstem anthranilsauren Natrium vorzulegen. Nach vier Stunden saugt man den gelben Niederschlag ab, wäscht mit verdünnter Schwefelsäure, dann mit $\mathrm{W}$ asser aus und trocknet

1) Auszug aus der Inaug.-Dissert. des Verf. (I. Teil), Dresden 1903.

2) Dies. Journ. [2] 33, 21 (1886).

3) Ber. 32. 259 . 
Thode: o-Amidobenzhydruzid 11. Abkomml. desselben. 93 schnell. Die so gewonnene Saiure ist rein, ihr Schmelzpunkt liegt bei $230^{\circ}$. Die beste Ausbeute, die erzielt wurde, betrug $80 \%$ der Theorie.

$50 \mathrm{~g}$ Isatosäure wurden mit $100 \mathrm{ccm}$ absolutem Alkohol gemischt, und $30 \mathrm{~g}$ einer 50 prozent. Hydrazinhydratlösung zugegeben. $\mathrm{Da}$ die Umsetzung unter Entwicklung von $\mathrm{CO}_{2}$ eine sehr energische ist, muB man zuerst in der Kälte operieren, weil man sonst durch plötzliches Überschäumen große Verluste erleidet. Sobald beim Erwärmen keine Gasentwicklung mehr stattindet, ist der ProzeB beendigt. Beim Erkalten scheidet sich das Hydrazid in nahezu reinem Zustande aus. Die Ausbeute schwankt zwischen $40 \%$ and $70 \%$, in einem Falle betrug sie $75 \%$ der Theorie.

Aus absolutem Alkohol kristallisiert die Verbindung in monoklinen Prismen, aus Chloroform in kurzen Nadeln, die den Schmelzp. $123^{\circ}$ zeigen. Sie ist leicht löslich in heißem Alkohol und Wasser, schwerer in Benzol und Äther.

Das o-A midobenzhydrazid läbt sich auch, jedoch weniger vorteilhaft, durch Einwirkung von Anthranilsäuremethylester auf Hydrazinhydrat gewinnen. $6 \mathrm{~g}$ des reinen Esters wurden im Ölbade 8 Stunden lang mit $4 \mathrm{~g}$ der 50 prozent. Hydrazinhydratlösung auf $150^{\circ}$ erhitzt. Die Ausbeute betrug nur $10 \%$ der Theorie. Die aus absolutem Alkohol umkristallisierte Verbindung schmolz bei $123^{\circ}$ und zeigte durch ihr ganzes Verhalten ihre Identität mit dem o-Amidobenzhydrazid.

$0,238 \mathrm{~g}$ Substanz gaben $0,483 \mathrm{~g} \mathrm{CO}_{2}$ und $0,1286 \mathrm{~g} \mathrm{H}_{2} \mathrm{O}$.

$0,1218 \mathrm{~g}$ Substanz gaben $29,3 \mathrm{ccm} \mathrm{N}$ bei $12^{\circ}$ und $740 \mathrm{~mm}$ Drack.

$\begin{array}{cccc}\text { Berechnet für } & \mathrm{C} & \mathbf{H} & \mathrm{N} \\ \mathrm{C}_{7} \mathrm{H}_{8} \mathrm{~N}_{8} \mathrm{O}: & 55,6 & 5,9 & 27,7 \% \\ \text { Gefunden: } & 55,3 & 6,0 & 27,7, \ldots\end{array}$

Salzsaures 0-A midobenzhydrazid, $\mathrm{C}_{8} \mathrm{H}_{4} \backslash{ }_{\mathrm{CO} . \mathrm{NH} . \mathrm{NH}_{2} . \mathrm{HCl}}^{\mathrm{NH}_{2} \cdot \mathrm{HCl}}$

Durch Einleiten von $\mathrm{HCl}$ in die abgekühlte alkoholische Lösung des Hydrazids fällt das Salz momentan aus und die Lösung erstarrt bald zu einem amorphen Brei. Aus ver- 
94 Thode: 0-Amidobenzhydrazid u. Abkomml, desselben.

dünntem Alkobol umkristallisiert und im Exsikkator getrocknet, wurde es analysiert: $31,6 \% \mathrm{Cl}$.

0,3102 g Substanz gaben $0,3349 \mathrm{~g} \mathrm{AgCl}=30,7 \% \mathrm{Cl}$; berechnet

Benzoisopyrazolon,<smiles>[CH2][C@H]1N[NH2+]O1</smiles>

Erhitzt man das 0-Amidobenzhydrazid auf $200^{\circ}$, so ist Entweichen von Ammoniak deutlich bemerkbar. Sobald die Gasentwicklung aufhört, läßt man erkalten und kristallisiert die geschmolzene Masse aus Alkohol aus. Der neue Körper bildet schöne Prismen, deren Schmelzpunkt bei $206^{\circ}$ liegt. Auch durch Kochen der mit Wasser verdünnten alkoholischen Lösung des Hydrazids tritt eine langsame, aber vollständige Umwandlung in die gleiche Verbindung ein. Zu ihrer Charakterisierung wurde, außer der Analyse, ihr Molekulargewicht nach der Eyckmanschen Methode bestimmt.

$0,0965 \mathrm{~g}$ Substanz wurden in 11,18 $\mathrm{g}$ Phenol gelöst. Die 0,863prozentige Lösung zeigte eine Erniedrigung ibrea Tefrierpunktes um $0,371^{\circ}$.

$\begin{array}{cc}\text { Berechnet: } & \text { Gefundeu: } \\ M=134 & 174 .\end{array}$

Die Analyse dieser nach mehrmaligem Umkristallisieren reinen Verbindung bot ganz besondere Schwierigkeiten; ihre Schwerverbrennlichkeit ist ungewöhnlich groß, wie die Analysenergebnisse deutlich erkennen lassen (s. u.). Nur durch inniges Mischen der gepulverten Substanz mit feinem Kupferoxyd waren brauchbare Resultate zu erhalten.

Das Silbersalz konnte wegen seiner groben Zersetzbarkeit nicht analysiert werden; bessere Erfolge waren mit dem Kupferdoppelsalz zu verzeichnen. Fügt man zur heißen Lösung des Benzoisopyrazolons eine konzentrierte Lösung von Kupfersulfat, so schlägt die blaue Farbe in eine grüne um. Beim Erkalten scheiden sich kleine, grüne Nädelchen des Kupferdoppelsalzes aus, deren Analysen unten mitgeteilt sind. 
Thode: 0-Amidobenzhydrazid u. Abkömml. desselben. 95

\section{Analysenergebnisse.)}

Benzoisopyrazolon: $\mathrm{C}_{7} \mathrm{H}_{8} \mathrm{~N}_{2} \mathrm{O}$.

I. $0,1275 \mathrm{~g}$ Substanz gaben $0,2633 \mathrm{~g} \quad \mathrm{CO}_{2}$ und $0,0585 \mathrm{~g} \mathrm{H}_{2} \mathrm{O}$.

II. $0,1096 \mathrm{~g}$ Substanz gaben $0,238 \mathrm{~g} \mathrm{CO}_{2}$ und $0,0519 \mathrm{~g} \mathrm{H}_{8} \mathrm{O}$.

III. $0,2719 \mathrm{~g}$ Substanz gaben $0,607 \mathrm{~g} \mathrm{CO}$ und $0,126 \mathrm{~g} \mathrm{H}_{2} \mathrm{O}$.

IV. $0,1076 \mathrm{~g}$ Substanz gaben $0,2402 \mathrm{~g} \mathrm{CO}_{2}$ und $0,0582 \mathrm{~g}_{2} \mathrm{O}$.

V. $0,361 \mathrm{~g}$ Substanz gaben $0,8095 \mathrm{~g} \mathrm{CO}_{2}$ und $0,1655 \mathrm{~g} \mathrm{H}_{\mathrm{z}} \mathrm{O}$.

VI. $0,0838 \mathrm{~g}$ Substanz gaben $0,1924 \mathrm{~g} \mathrm{CO}_{2}$ und $0,0415 \mathrm{~g} \mathrm{H}_{2} \mathrm{O}$.

VII. $0,121 \mathrm{~g}$ Substanz gaben $20,8 \mathrm{~cm} N$ bei $14^{\circ}$ und $748 \mathrm{~mm}$.

VIII. $0,097 \mathrm{~g}$ Substanz gaben $16,8 \mathrm{ccm} N$ bei $16^{\circ}$ und $758 \mathrm{~mm}$.

IX. $0,1067 \mathrm{~g}$ Substanz gaben $19,2 \mathrm{ccm} \mathrm{N}$ bei $15^{\circ}$ und $752 \mathrm{~mm}$.

X. 0,1612 g Substanz gaben $28,3 \mathrm{ccm} N$ bei $17^{\circ}$ und $762 \mathrm{~mm}$.

$\begin{array}{cccc}\text { Berechnet für } & \mathrm{C} & \mathrm{H} & \mathrm{N} \\ \mathrm{C}_{7} \mathrm{H}_{6} \mathrm{~N}_{2} \mathrm{O}: & 62,6 & 4,4 & 20,8 \% \\ \text { Gefunden: } & & & \\ \text { I. } & 56,4 & 5,0 & - \\ \text { II. } & 59,2 & 5,2 & - \\ \text { III. } & 60,5 & 5,0 & - \\ \text { IV. } & 60,8 & 6,0 & - \\ \text { V. } & 61,1 & 5,0 & - \\ \text { VI. } & 62,5 & 5,5 & - \\ \text { VII. } & - & - & 19,9 \% \\ \text { VIII. } & - & - & 20,1 \% \\ \text { IX. } & - & - & 20,8 \% \\ \text { X. } & - & - & 20,3 \%\end{array}$

Nur die Ergebnisse von VI. und IX. sind für $\mathbf{C}$ und $\mathbf{N}$ völlig genügend zu nennen; von den H-Bestimmungen lassen sich die unter I., III. und V. angeführten verwerten.

Die Zusammensetzung des Kupferdoppelsalzes führt ebenfalls zu der obigen Formel des Benzoisopyrazolons.

Analysenergebnisse des Doppelsalzes,

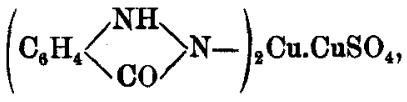

(getrocknet bei $110^{\circ}$ ).

I. 0,7582 g Substanz gaben 0,3658 g $\mathrm{BaSO}_{4}$.

II. 0,2256 g Substanz gaben $0,2798 \mathrm{~g} \mathrm{CO}_{2}$ und $0,0611 \mathrm{~g} \mathrm{H}_{2} \mathrm{O}$. 
96 Thode: 0-Amidobenzhydrazid u. Abkomml. desselben.

III. 0,2112 g Substanz gaben $0,2615 \mathrm{~g} \mathrm{CO}_{2}$ und $0,0518 \mathrm{~g} \mathrm{H}_{2} \mathrm{O}$.

IV. $0,1639 \mathrm{~g}$ Substanz gaben $17,2 \mathrm{ccm} N$ bei $18^{\circ}$ and $766 \mathrm{~mm}$.

V. $0,2504 \mathrm{~g}$ Substanz gaben $0,0819 \mathrm{~g} \mathrm{CuO}$

VI. 0,1952 g Substanz gaben 0,0624 g CuO.

$\begin{array}{cccccc}\begin{array}{c}\text { Berechnet für } \\ \mathrm{C}_{14} \mathrm{H}_{10} \mathrm{~N}_{2} \mathrm{O}_{\mathbf{6}} \mathrm{Cu}_{2} \mathrm{~S}:\end{array} & \mathrm{C} & \mathrm{H} & \mathrm{N} & \mathrm{S} & \mathrm{Cn} \\ \text { Gefunden: } & 34,4 & 2,0 & 11,4 & 6,5 & 25,8 \% \\ \text { I. } & & & & & \\ \text { II. } & - & - & - & 6,6 & - \\ \text { III. } & 33,0 & 2,7 & - & - & - \\ \text { IV. } & 33,7 & 2,7 & - & - & - \\ \text { V. } & - & - & 12,1 & - & -\overline{0} \% \\ \text { VI. } & - & - & - & - & 26,0 \% \\ & - & - & - & - & 25,4 \%\end{array}$

Nach ihrer Entstehungsweise und Zusammensetzung ist die obige Verbindung als Benzoisopyrazolon,<smiles>[CH2+][C@H]1NN[O+]O1</smiles>

aufgefaBt worden. E. Fischer ${ }^{1}$ ) hat nun aus o-Hydrazidobenzoësäure durch Erhitzen deren Anhydrid gewonnen und in derselben Weise formuliert.

Die beiden Körper sind aber sicherlich von einander verschieden. Das obige "Benzoisopyrazolon" schmilzt bei $206^{\circ}$, das Anhydrid Fischers erst oberhalb $220^{\circ}$ unter fortschreitender Zersetzung; letzteres liefert mit Essigsäureanhydrid ein Diacetylderivat, ersteres wird unter gleichen Umständen nicht in ein solches übergeführt.

Vielleicht ist das Anhydrid Fischers folgendermaBen konstituiert:<smiles>Oc1nnc(-c2ccccc2)[nH]1</smiles>

1) Ber. 13, 681: Ann. Chem. 212, 333. 
Thode: 0-Amidobenzhydrazid u. Abkömml. desselben 97

Seine Entstehung würde dann der des Isatins aus oA midobenzoylkarbonsäure entsprechen:

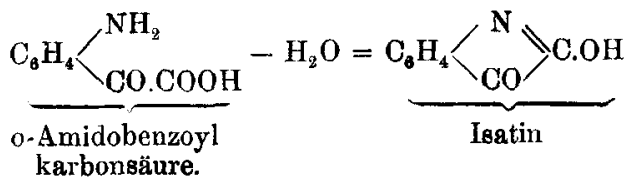

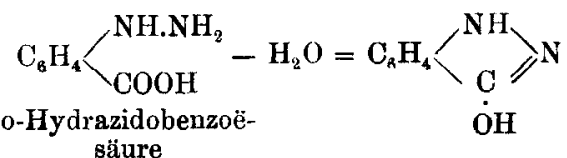

Benzal-0-amidobenzhydrazid,

$$
\mathrm{C}_{6} \mathrm{H}_{4}<{ }_{\mathrm{CO} . \mathrm{NH} . \mathrm{N}-\mathrm{CH} . \mathrm{C}_{6} \mathrm{H}_{8}}^{\mathrm{NH}_{3}}
$$

Versetzt man die alkoholische Lösung des 0-Amidobenzhydrazids mit der berechneten Menge Benzaldehyd und kocht dieselbe, so erfolgt die Kondensation glatt nach der Gleichung:

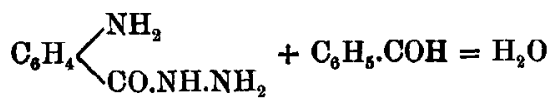

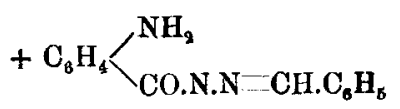

Die Nädelchen des Benzalproduktes waren gelb, wurden aber durch mehrmaliges Kochen ihrer Lösung mit Tierkohle fast farblos. Das Benzal-o-amidobenzhydrazid ist in warmem Alkohol und Benzol sehr leicht löslich, schwerer in Wasser und Ligroin; es schmilzt bei $195^{\circ}$.

Durch Kochen mit verdünnten Säuren zerfällt es glatt in seine Komponenten.

$0,1240 \mathrm{~g}$ Substanz gaben $18,7 \mathrm{ccm} \mathrm{N}$ bei $14^{\circ}$ und $748 \mathrm{~mm}$ Druck $=17,5 \% \mathrm{~N}$.

Berechnet: $17,5 \%$. 
98 Thode: o-Amidobenzhydrazid u. Abkömml. desselben.

A cetyl-Benzal-0-amidobenzhydrazid,

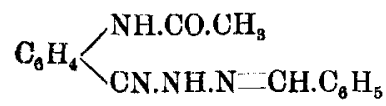

Wird die Benzalverbindung mit etwas mehr als der berechneten Menge Essigsäureanhydrid bis zum Sieden erhitzt, so bildet sich das Acetylderivat. Beim Erkalten erstarrt die ganze Masse zum Kristallbrei. Die Acetylverbindung ist schwer löslich in Alkohol und Eisessig, unlöslich in Äther und Wasser. Sie kristallisiert aus verdünnter Essigsäure in prismatischen Tafeln, die bei $180^{\circ}$ unter Gasentwicklung schmelzen.

$0,1138 \mathrm{~g}$ Substanz gaben $15,1 \mathrm{ccm} N$ bei $18^{\circ}$ und $752 \mathrm{~mm}$ Druck $=15,1 \% \mathrm{~N}$.

Berechnet: $14,9 \%$.

Diacetonylamidobenzhydrazid,<smiles>CCNC(=O)NC(=O)NN=C(C)C</smiles>

o-Amidobenzhydrazid löst sich in überschüssigem Aceton unter Wärmeentwicklung auf. Beim Erkalten der Lösung scheiden sich schöne Nadelaggregate aus, und je nach der Menge Aceton erstarrt bald auch die ganze Masse. Nach folgender Gleichung ist Kondensation mit 2 Mol. Aceton eingetreten:

$$
\begin{aligned}
& \mathrm{C}_{6} \mathrm{H}_{4}\left\langle\begin{array}{l}
\mathrm{NH}_{8} \\
\mathrm{CO} . \mathrm{NH}-\mathrm{NH}_{2}
\end{array}+2 \mathrm{CH}_{8} \cdot{\mathrm{CO} . \mathrm{CH}_{3}}_{3}\right. \\
& =2 \mathrm{H}_{2} \mathrm{O}+\mathrm{C}_{8} \mathrm{H}_{4}<\begin{array}{l}
\mathrm{N}=\mathrm{C}\left(\mathrm{CH}_{8}\right)_{2} \\
\mathrm{CO} . \mathrm{NH} . \mathrm{N}-\mathrm{C}\left(\mathrm{CH}_{8}\right)_{2}
\end{array}
\end{aligned}
$$

Vielleicht ist auch folgende Formel<smiles>C=C1NOC(NCCC)C(=C)N1</smiles>

zu berücksichtigen. 
Thode: 0-Amidobenzhydrazid u. Abkomml. desselben. 99

Diese Verbindung scheidet sich völlig rein aus. Schmelzpunkt 244. Aus absolutem Alkohol kristallisiert sie in schönen Prismen. Sie ist in Alkohol und Benzol leicht löslich, in Aceton schwer löslich.

I. $0,077 \mathrm{~g}$ Substanz gaben $0,1907 \mathrm{~g} \mathrm{CO}_{2}$ und $0,053 \mathrm{~g} \mathrm{H}_{2} \mathrm{O}$. Druck.

II. $0,0973 \mathrm{~g}$ Substanz gaben $15,0 \mathrm{ccm} \mathrm{N}$ bei $13^{\circ}$ and $762 \mathrm{~mm}$

$\begin{array}{ccc} & \text { Berechnet für } \mathrm{C}_{18} \mathrm{H}_{17} \mathrm{~N}_{3} \mathrm{O}: & \text { Gefunden: } \\ \mathrm{C} & 67,5 & 67,5 \% \\ \mathrm{H} & 7,3 & 7,5 \% \\ \mathrm{~N} & 18,1 & 18,2 \%\end{array}$

Acetophenon-o-amidobenzhydrazid,<smiles>[NH3+]C(=O)NNC(=O)NCCc1ccccc1</smiles>

Die alkoholische Lösung äquimolekularer Mengen von Acetophenon und o-Amidobenzhydrazid wurde zum Sieden erhitzt, wobei die erwartete Reaktion eintrat. Das Produkt kristallisiert aus absolutem Alkohol in prismatischen Platten, die bei $165^{\circ}$ schmelzen. In siedendem Alkohol und Benzol ist es ziemlich leicht löslich, nicht so leicht löst es sich in Äther und sehr schwer in Wasser.

$0,1184 \mathrm{~g}$ Substanz gaben $17,7 \mathrm{ccm} N$ bei $13^{\circ}$ und $746 \mathrm{~mm}$ Druck $=17,1 \% \mathrm{~N}$.

Berechnet: $16,7 \%$.

Benzophenon-o-amidobenzhydrazid,<smiles>NC(N)C(=O)NN=C(c1ccccc1)c1ccccc1</smiles>

Hier erfolgte in alkoholischer Lösung keine Kondensation, dieselbe trat aber bald in glatter Weise ein beim direktem Erhitzen der beiden Verbindungen auf $180^{\circ}$.

Nach zweimaligem Umkristallisieren aus Alkohol hatten die Nadeln den Schmelzp. 157\%. Der Körper ist in kaltem Aceton, heißem Alkohol und Benzol leicht löslich, schwerer in Äther und fast unlöslich in Wasser. 
100 Thode: 0-Amidobenzhydrazid u. Abkömml. desselben $0,1624 \mathrm{~g}$ Substanz gaben $18,8 \mathrm{ecm} \mathrm{N}$ bei $13^{\circ}$ und $766 \mathrm{~mm}$ Druck $=13,4 \% \mathrm{~N}$.

Berechnet: $13,3 \%$

Ameisensäure und o-Amidobenzhydrazid.

Methenyl-o-amidobenzhydrazid,<smiles></smiles>

Eine Lösung von einem Molekül des Hydrazids in der berechneten Menge Ameisensäure wurde bis zum Sieden erhitzt und erkalten gelassen. Das Gemisch erstarrte, und aus absolutem Alkohol kristallisierten prismatische Nadeln, die schwach gefärbt waren und bei $204^{\circ}$ schmolzen.

Die Reaktion erfolgt unter Austritt von 2 Mol. Wasser:

$$
\begin{aligned}
& \mathrm{C}_{6} \mathrm{H}_{4}\left\langle\mathrm{NH}_{\mathrm{CO} . \mathrm{NH}-\mathrm{NH}_{2}}^{\mathrm{NH}_{2}}+\mathrm{HCOOH}=2 \mathrm{H}_{2} \mathrm{O}\right. \\
& +\mathrm{C}_{6} \mathrm{H}_{4} \backslash \stackrel{\mathrm{N}=\mathrm{CH}}{\mathrm{CO} . \mathrm{NH} . \mathrm{NH}_{8}}
\end{aligned}
$$

Das Produkt kann auch als Keto-2-amidodihydrochinazolin bezeichnet werden.

Diese Verbindung hat noch schwach reduzierende Eigenschaften. In heißem, absoluten Alkohol leicht löslich, schwerer löslich in Eisessig, Äther und Wasser.

0,5412 g Substanz gaben $1,1839 \mathrm{~g} \mathrm{CO}_{2}$ und $0,2087 \mathrm{~g} \mathrm{H}_{2} \mathrm{O}$.

$0,2346 \mathrm{~g}$ Substanz gaben $52,5 \mathrm{ccm} N$ bei $14^{\circ}$ und $750 \mathrm{~mm}$ Druck.

$\begin{array}{ccc} & \text { Berechnet für } \mathrm{C}_{8} \mathrm{H}_{7} \mathrm{~N}_{3} \mathrm{O}: & \text { Gefunden: } \\ \mathrm{C} & \mathbf{5 9 , 6} & 59,6 \% \\ \mathrm{H} & 4,3 & 4,2, \% \\ \mathrm{~N} & 26,08 & 25,8 \%\end{array}$

Benzaldehyd und Methenyl-0-amidobenzhydrazid.

Um die freie, an Stickstoff gebundene Amidogruppe im Molekül der Verbindung nachzuweisen, wurde eine Konden- 
Thode: 0-Amidobenzhydrazid u. Abkömml. desselben. 101 sation mit Benzaldehyd versucht, der damit auch leicht nach der Gleichung:

$$
\begin{gathered}
\mathrm{C}_{6} \mathrm{H}_{4} \backslash \begin{array}{l}
\mathrm{N}=\mathrm{CH} \\
\mathrm{CO} \cdot \mathrm{NH} . \mathrm{NH}_{2}
\end{array}+\mathrm{C}_{6} \mathrm{H}_{6} \cdot \mathrm{COH}=\mathrm{H}_{2} \mathrm{O} \\
+\mathrm{C}_{6} \mathrm{H}_{4} \backslash \begin{array}{l}
\mathrm{N}=\mathrm{CH} \\
\mathrm{CO} . \mathrm{NH} . \mathrm{N}=\mathrm{CH} \cdot \mathrm{C}_{6} \mathrm{H}_{5}
\end{array}
\end{gathered}
$$

reagierte.

Die Blättchen, die aus absolutem Alkohol auskristallisieren, sind in Wasser und Benzol schwer, leicht in heißem Alkohol löslich und schmelzen bei $129^{\circ}$. Auch hier tritt beim Kochen mit verdünnten Säuren leicht die Abspaltung von Benzaldehyd ein, wie bei den analogen Benzalderivaten.

$0,2160 \mathrm{~g}$ Substanz gaben $31,0 \mathrm{ccm} N$ bei $13^{\circ}$ und $762 \mathrm{~mm}$ Druck $=16,9 \% \mathrm{~N}$.

Berechnet: $16,8 \%$.

Salicylaldehyd und Methenylverbindung,<smiles></smiles>

Werden äquimolekulare Mengen dieser beiden zusammen auf $250^{\circ}$ erhitzt, so bildet sich die obige Verbindung, die in langen, feinen, sich sehr leicht zusammenballenden Nadeln kristallisiert und bei $205^{\circ}$ scbmilzt; sie ist wenig löslich in Wasser, Ligroïn, Benzol und Äther, leicht in Alkohol.

$0,1286 \mathrm{~g}$ Substanz gaben $12 \mathrm{ccm} \mathrm{N}$ bei $22^{\circ}$ und $758 \mathrm{~mm}$ Druck $=1 \tilde{0}, 8 \% \mathrm{~N}$.

Berechnet: $15,8 \%$.

Ameisensäure und o-Amidobenzphenylhydrazid:

1-Keto-2-phenylamidodihydrochinazolin,

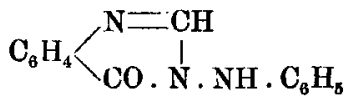

Nach E. von Meyer und Bellmann ${ }^{1}$ ) wurde das oAmidobenzphenylhydrazid durch Wechselwirkung von Phenyl-

1) Dies, Journ. [2] 33, 21. 
102 Thode: 0-Amidobenzhydrazid u. Abkömml. desselben. hydrazin und Isatosäure dargestellt. Äquimolekulare Mengen reagieren in alkoholischer Lösung bei $60^{\circ}$. In fast quantitativer Ausbeute erhält man das Phenylhydrazid in schönen Prismen, die, aus absolutem Alkohol umkristallisiert, bei $170^{\circ}$ schmelzen.

Hauptbedingung zur Gewinnung des Chinazolinderivates ist die Anwendung von ganz wasserfreier Ameisensäure. Das Reaktionsgemisch wird längere Zeit siedend erhalten, und nach dem Erkalten kristallisiert man die erstarrte Masse aus absolutem Alkohol um.

Die Methenylverbindung kristallisiert aus Alkohol in schönen Prismen, die den Schmelzp. $140^{\circ}$ besitzen. In kaltem und siedendem Wasser ist der Körper schwer löslich, etwas in Äther. In Benzol löst er sich leicht auf; auf Zusatz von Ligroïn fällt er in schönen, weißen Blättchen aus, die denselben Schmelzpunkt haben.

$0,1255 \mathrm{~g}$ Substanz gaben $19,1 \mathrm{ccm} \mathrm{N}$ bei $14^{\circ}$ und $758 \mathrm{~mm}$ Druck $=17,8 \% \mathrm{~N}$.

Berechnet: $17,7 \%$.

Einwirkung von salpetriger Säure auf das o-Amidobenzhydrazid.

In der Hoffnung, das o-A midobenzazid,<smiles>N[14C](=O)c1ccccc1</smiles>

zu erhalten, wurde salpetrige Säure auf das Hydrazid einwirken gelassen; jedoch erwies sich die naheliegende Befürchtung, daß die orthoständige Amidogruppe in Reaktion treten werde, als begründet. Statt des Azids entstand eine Verbindung, die ich mit der von Weddige ${ }^{1}$ ) unter dem Namen Benzazinid<smiles>NN=NC(=O)c1ccccc1</smiles>

beschriebenen für identisch halte.

1) Dies. Journ. [2] 35, 262. 
Thode: 0-Amidobenzhydrazid u. Abkömml. desselben. 103

Die Reaktion wurde mit dem salzsauren Salz des Hydrazids in wäbriger Lösung und $\mathrm{NaNO}_{2}$ durchgeführt. Um möglichst gute Ausbeute zu erzielen, darf man die Temperatur nicht über $+5^{0}$ steigen lassen. Der Körper scheidet sich sofort aus und wird am besten aus Eisessig umkıistallisiert. In Wasser und Benzol ist er schwer löslich. Beim längeren Stehen an Licht und Luft tritt langsam Zersetzung ein. Derselbe Versuch wurde in essigsaurer Lösung vorgenommen, ergab aber kein anderes Resultat.

$0,109 \mathrm{~g}$ Substanz gaben $0,226 \mathrm{~g} \mathrm{CO}_{2}$ und $0,0364 \mathrm{~g} \mathrm{H}_{2} \mathrm{O}$.

$0,0762 \mathrm{~g}$ Substanz gaben $19,2 \mathrm{ccm} \mathrm{N}$ bei $15^{\circ}$ und $747 \mathrm{~mm}$ Druck.

$\begin{array}{ccc} & \text { Berechnet für } \mathrm{C}_{\mathbf{7}} \mathrm{H}_{8} \mathrm{~N}_{3} \mathrm{O}: & \text { Gefunden: } \\ \mathrm{C} & \mathbf{5 7 , 1} & \mathbf{5 6 , 5} \% \\ \mathrm{H} & \mathbf{3 , 4} & \mathbf{3 , 7} \% \\ \mathrm{~N} & 28,5 & \mathbf{2 8 , 8}, .\end{array}$

Salpetrige Säure und 0-Amidobenzphenylhydrazid.

Durch Einwirkung von salpetriger Säure auf das Phenylhydrazid wurde die Bildung einer Verbindung von folgender Formel erwartet:

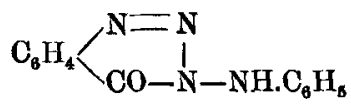

Auch hier wurde die Reaktion in salzsaurer Lösung vorgenommen, die wäßrige Lösung des Natriumnitrits wird langsam eingetragen und die Temperatur bei $0^{0}$ erhalten. Der entstehende amorphe Körper läßt sich nicht völlig reinigen, da er aus keinem Lösungsmittel kristallisiert. Er zersetzt sich bei $78^{\circ}$ und verpufft leicht beim schnellen Erhitzen.

Am besten stimmen die Analysenergebnisse auf das Nitrosoderivat des o-Amidobenzphenylhydrazids:<smiles>CCONNNC(=O)OCC</smiles>

$0,1065 \mathrm{~g}$ Substanz gaben $0,2325 \mathrm{~g} \mathrm{CO}_{2}$ und $0,0495 \mathrm{~g} \mathrm{H}_{2} \mathrm{O}$.

$0,0972 \mathrm{~g}$ Substanz gaben $18,1 \mathrm{ccm} N$ bei $16^{\circ}$ und $756 \mathrm{~mm}$ Druck. 
104 Thode: 0-Amidobenzhydrazid u. Ablömml. desselben.

$\begin{array}{ccc} & \text { Berechnet für } \mathrm{C}_{19} \mathrm{H}_{12} \mathrm{~N}_{4} \mathrm{O}_{2}: & \text { Gefunden: } \\ \mathrm{C} & 60,9 & 59,5 \% \\ \mathrm{H} & 4,7 & 5,1 \% \\ \mathrm{~N} & 21,8 & 21,6 \%\end{array}$

Die Verbindung wird von heißem Alkohol leicht zersetzt. Es entweicht Stickstoff und nach dem Erkalten fällt eine stickstoff ärmere Verbindung in feinen Nädelchen aus, deren Schmelzpunkt bei $206^{\circ}$ liegt. Die Werte der Analysen weisen auf eine Verbindung $\mathrm{C}_{13} \mathrm{H}_{12} \mathrm{~N}_{2} \mathrm{O}$ hin; das so zusammengesetzte, bekannte Benzoylphenylhydrazin ist obiger Körper nicht.

$0,1505 \mathrm{~g}$ Substanz gaben $0,3997 \mathrm{~g} \mathrm{CO}_{2}$ und $0,0740 \mathrm{~g} \mathrm{H}_{2} \mathrm{O}$.

$0,1413 \mathrm{~g}$ Substanz gaben $16,4 \mathrm{ccm} \mathrm{N}$ bei $21,5^{\circ}$ und $760 \mathrm{~mm}$ Druck.

$\begin{array}{ccc} & \text { Berechnet für } \mathrm{C}_{13} \mathrm{H}_{12} \mathrm{~N}_{2} \mathrm{O}: & \text { Gefunden: } \\ \mathrm{C} & \mathbf{7 3 , 5} & \mathbf{7 2 , 4} \% \\ \mathrm{H} & 5,6 & 5,4 \% \\ \mathrm{~N} & 13,2 & 13,1 \% \text {. }\end{array}$

